\title{
Human Ability to Recognize Kin Visually Within Primates
}

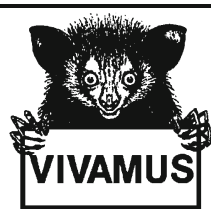

\author{
Alexandra Alvergne • Elise Huchard • \\ Damien Caillaud • Marie J. E. Charpentier • \\ Joanna M. Setchell • Charlène Ruppli • \\ Delphine Féjan • Laura Martinez • \\ Guy Cowlishaw $\cdot$ Michel Raymond
}

Received: 13 May 2008 / Accepted: 7 September 2008 /

Published online: 31 January 2009

(C) Springer Science + Business Media, LLC 2009

\begin{abstract}
The assessment of relatedness is a key determinant in the evolution of social behavior in primates. Humans are able to detect kin visually in their own species using facial phenotypes, and facial resemblance in turn influences both prosocial behaviors and mating decisions. This suggests that cognitive abilities that allow facial kin detection in conspecifics have been favored in the species by kin selection. We investigated the extent to which humans are able to recognize kin
\end{abstract}

\footnotetext{
A. Alvergne $(\bowtie) \cdot$ E. Huchard $\cdot$ D. Caillaud $\cdot$ C. Ruppli $\cdot$ D. Féjan $\cdot$ M. Raymond Institute of Evolutionary Sciences, University of Montpellier II and CNRS, 34095 Montpellier Cedex 5, France

e-mail: alexandra.alvergne@um2.fr

E. Huchard

e-mail: ehuchard@gmail.com

D. Caillaud

Department of Primatology, Max Planck Institute for Evolutionary Anthropology, 04103 Leipzig, Germany
}

\section{J. E. Charpentier}

Centre d'Ecologie Fonctionnelle et Evolutive UMR 5175, CNRS, 34293 Montpellier Cedex 5, France

M. J. E. Charpentier · J. M. Setchell

Centre International de Recherches Médicales, BP 769, Franceville, Gabon

J. M. Setchell

Evolutionary Anthropology Research Group, Department of Anthropology, Durham University,

Durham DH1 3HN, UK

L. Martinez

Language and Intelligence Section, Primate Research Institute, Kyoto University, Inuyama,

Aichi 484-8506, Japan

G. Cowlishaw

Institute of Zoology, Zoological Society of London, London NW1 4RY, UK 
visually by asking human judges to assess facial resemblance in 4 other primate species (common chimpanzees, western lowland gorillas, mandrills, and chacma baboons) on the basis of pictures of faces. Humans achieved facial interspecific kin recognition in all species except baboons. Facial resemblance is a reliable indicator of relatedness in at least chimpanzees, gorillas, and mandrills, and future work should explore if the primates themselves also share the ability to detect kin facially.

Keywords facial resemblance $\cdot$ humans $\cdot$ interspecific visual kin recognition

\section{Introduction}

Faces are pivotal for social interactions among primates (Niedenthal et al. 2000). Researchers have observed the ability to identify conspecific faces on the basis of pictures in many species, including chimpanzees (Pan troglodytes: Parr et al. 1998), long-tailed macaques (Macaca fascicularis: Dasser 1987), Japanese macaques (Macaca fuscata: Tomonaga 1994), and rhesus macaques (Macaca mulatta: Parr et al. 1999; Pascalis and Bachevalier 1998). Systems of face recognition present some similarities between human and nonhuman primates. For instance, human newborns and macaque infants exhibit a preference for face-like stimuli vs. object-like stimuli, suggesting that face processing is partly experience-independent (Johnson et al. 1991, Sugita 2008). In addition, a species-specific effect, defined as an advantage in recognizing faces of an individual's own species, or conspecific faces, occurs in various species, including humans, rhesus macaques (Dufour et al. 2004; Pascalis and Bachevalier 1998), tonkean macaques (Macaca tonkeana), and brown capuchins (Cebus apella: Dufour et al. 2006)

Facial recognition may be achieved via the selection of a face recognition system that is highly dependent on exposure (Dufour et al. 2006; Pascalis and Bachevalier 1998). Indeed, the facial recognition system is plastic in at least chimpanzees and humans. Chimpanzees raised by humans from a juvenile age show a reverse speciesspecific effect, preferring human faces (Tanaka 2003). In humans, face recognition is better for individuals of the same ethnic group, a phenomenon referred to as the other-race effect (Lindsay et al. 1991; O'Toole et al. 1994). Interestingly, if the other race effect develops during infancy (Kelly et al. 2007), the face recognition system remains sufficiently plastic that the effect may be reversed by subsequent experience with new types of faces (Sangrigoli et al. 2005).

Though humans can accurately identify their conspecifics via the face, they can also recognize kinship relationships via facial phenotype. The detection of relatedness is performed through the assessment of facial similarities between individuals, and humans correctly associate either siblings or parents and offspring (Alvergne et al. 2007; Maloney and Dal Martello 2006). One can propose 2 hypotheses to explain the ability to recognize kinship associations between others than themselves. First, it could reflect a byproduct of the ability to recognize its own kin in the context of kin selection theories. In humans, the ability to detect one's own kin facially can be associated with important fitness benefits because facial resemblance exerts a positive effect on paternal investment and cooperation and a negative effect on attractiveness in a mating context (DeBruine 2002, 2004a, b, 2005; DeBruine et al. 2008; Little et al. 2008; Platek et al. 2003). 
A second possibility is that the recognition of kin relationships between other individuals, especially other members of a group, is advantageous, at least for animals living in complex social environments. Empirical studies support the latter hypothesis: in several primate species with matrilineal dominance hierarchies, females can categorize signallers on the basis of their matriline, either vocally or facially (Java monkeys: Dasser 1988; vervets: Cheney and Seyfarth 1982; macaques: Rendall et al. 1996, chacma baboons: Bergman et al. 2003; Cheney and Seyfarth 1999), and adjust their social interactions to kinship relationships between other individuals: for example, female baboons that were threatened by another female avoid their aggressor's close relatives for a longer period than any other unrelated individual (Wittig et al. 2007). However, whether the apparent recognition is mediated by familiarity, via patterns of affiliation and proximity, or by phenotypic similarities remains unknown.

There is a possibility that primates share a similar system to detect kinship relationships. For instance, from an intraspecific perspective, chimpanzees, just as humans (Alvergne et al. 2007; Maloney and Dal Martello 2006), are able to detect mother-son pairs in conspecifics on the basis of facial similarities (Parr and de Waal 1999), although the experimental setting has been criticized because of framing biases (Vokey et al. 2004). In addition, from an interspecific perspective, a first and only study has shown that humans correctly assign mother-offspring pairs in chimpanzees using facial photographs (Vokey et al. 2004). The result may be explained by the proximity of facial features between chimpanzees and humans, allowing the human facial kin-recognition system to operate in related species.

We investigated the ability of humans to recognize kin visually via facial comparisons in 4 nonhuman primate species: 2 apes (common chimpanzee, western lowland gorillas) and 2 cercopithecines (mandrills and chacma baboons). Such a protocol specifically aims to compare the human ability to detect facial resemblance in primate species that share variable degrees of phylogenetic proximity, and to use the human eye as a tool to detect the potential existence of facial kin resemblance in the species, for which, except in chimpanzees, researchers have not tested.

\section{Methods}

\section{Procedure}

We randomly recruited 618 human judges in public places on a voluntary basis. Forty-three percent of judges were men $(43 \%, n=266)$ and $57 \%$ were women $(n=$ 352 ), 15-60 yr of age (mean \pm SD: $36.3 \pm 9.9$ ). Each judge performed a series of tests. During each test, we presented a picture depicting the face of a referent individual to the human judge along with the faces of 3 other individuals (Fig. 1). Among the 3 individuals, 1 was related to the referent individual. We randomized both the position on the screen and the order of presentation of nonreferent pictures for each trial. For each of the referent pictures, we randomly drew pictures of unrelated individuals from the set of pictures not used as referents. We presented no picture $>1$ time to each judge. The judges performed kin recognition tests for 1 species only. We instructed them to take as much time they needed, and they were unaware that 


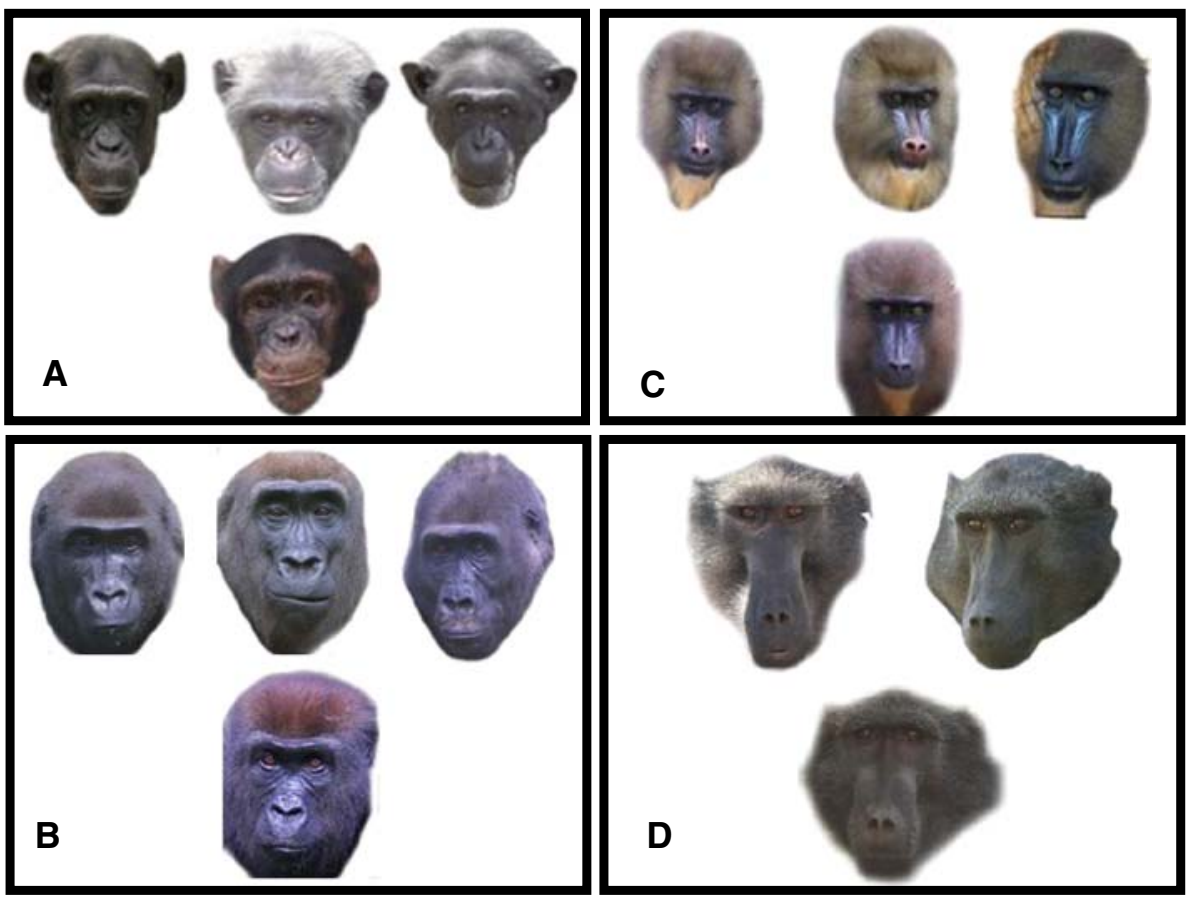

Fig. 1 Interspecific facial kin recognition test. We asked human judges to 1) look at the face of the referent individual presented below and to (2) choose which picture was most likely to portray its female relative among the pictures proposed above. We randomized the order of the referent individuals, the position of relatives, as well as the choice of nonrelatives. A given judge viewed each picture only once, and we presented each judge to 1 species only. (A) Facial kin recognition test using chimpanzee faces. The correct choice is the left picture. (B) Facial kin recognition test using gorilla faces. The correct choice is the middle picture. (C) Facial kin recognition test using mandrill faces. The correct choice is the right picture. (D) Facial kin recognition test using baboon faces. The correct choice is the right picture.

we automatically recorded their answer time. We assessed facial resemblance only to female relatives who shared a high degree of relatedness, i.e., $r \sim 0.5$, with the referent individuals.

\section{Sources of Facial Photographs}

Huchard collected facial photographs of chacma baboons (Papio ursinus) in a wild population living in Tsaobis Leopard Park, Central Namibia (Cowlishaw 1999). Beginning in 2000, baboons were captured to gather biological samples and morphological measurements. We thus estimated age according to known tooth eruption schedules (Kahumbu and Eley 1991) and molar wear patterns ( $c f$. PhillipsConroy et al. 2000). We performed tests on 12 referent individuals (3 males and 9 females) 7-19 yr of age (mean $\pm \mathrm{SD}$ : 11.4 \pm 4.2 ). We proposed possible referentrelated females of 2.4-16.2 yr including relatives and nonrelatives (mean $\pm \mathrm{SD}: 9.9 \pm$ 3.1). Setchell collected facial photographs of mother-daughters pairs in mandrills (Mandrillus sphinx) in a semifree-ranging colony housed at CIRMF, Gabon. We also obtained pictures of additional adult females from the zoo of Peaugres, France (with 
the permission of Mélanie Pignorel, DVM) to increase the number of available nonrelated individuals. We knew mandrill ages from colony records. We proposed 10 females $2.2-7.5 \mathrm{yr}$ of age (mean $\pm \mathrm{SD}: 4.9 \pm 1.9$ ) as referent individuals. The nonreferent individuals, including related and nonrelated females, were 3-22 yr old (mean \pm SD: 11.2 \pm 6.6 ). Caillaud collected facial photographs of mother-offspring pairs in western lowland gorilla (Gorilla gorilla gorilla) from wild individuals visiting the Lokoue clearing in Odzala-Kokoua National Park, Republic of the Congo (Caillaud et al. 2006). We included 9 unweaned gorilla infants, 1-3 yr of age, used as referent individuals, and their mothers. We did not know the sex of the unweaned infants and the exact age of nonreferent individuals. We obtained photographs of mother-offspring pairs in chimpanzee faces from 2 different sources: Martinez collected 6 pairs of the western chimpanzee subspecies (Pan troglodytes verus) at the Primate Research Institute of Kyoto University and from Chausuyama Zoological Park, Japan (with the permission of Tomomi Ochiai, GAIN network and Tetsuro Matsuzawa); 3 pairs of central chimpanzee subspecies (P. t. troglodytes) came from the zoological park of St Martin la Plaine, France (with the permission of Sébastien Virth). We knew the age of the chimpanzees from zoo records. The chimpanzee sample thus comprises 9 offspring used as referent individuals, including 4 males and 5 females $4-24$ yr of age (mean \pm SD: $7.6 \pm 0.9$ ). The nonreferent individuals (mothers and nonrelated females) were 18-39 yr of age (mean \pm SD: 27.7 \pm 7.6 ). We processed all photographs using Adobe Photoshop v. 7.0 to standardize contrast and brightness, and to turn all backgrounds white.

\section{Relatedness Assessment}

For chimpanzees, we used mother-offspring pairs established via zoo pedigree records. For gorillas, we established mother-offspring pairs from behavioral observations and patterns of maternal care. For mandrills, we used mother-daughters pairs for which the coefficient of relatedness retrieved from pedigree records was 0.5 . For baboons, we calculated pairwise relatedness $(r)$, between all pairs of related females, using individual genotypes at 16 microsatellite loci (mean number of loci genotyped $\pm \mathrm{SEM}=16 \pm 0$; details on microsatellite typing are in Knapp et al. in prep). Specifically, we used a triadic likelihood (TL) estimator of relatedness per the method developed by Wang (2007). In this method, each individual in the sampled population is used as a reference when estimating the coefficient of relatedness between 2 other individuals, thus using triads rather than dyads of individuals to reduce the chance that genes identical by state are mistakenly inferred as identical by descent when calculating pairwise estimates of relatedness within dyads (Wang 2007). Using TL relatedness coefficients and a larger sample derived from the same population $(n=209)$, pairwise relatedness calculated by averaging TL pairwise coefficient of relatedness for all pairs of individuals of the population $(n=21,945)$ ranged from 0 to 0.603 (median $=0.012$, mean \pm SD: $0.063 \pm 0.10$ ). The average value of pairwise relatedness between 56 mother-offspring pairs known from behavioral observations was $0.44 \pm 0.08$, which shows that this absolute relatedness estimate is slightly below theoretical expectations $(0.5)$ due to the high background relatedness in the sample, which is typical of savannah baboon groups as a result of female philopatry and male-biased dispersal (Altmann et al. 1996). We matched pairs of 
females for which the pairwise coefficient of relatedness exceeded the average pairwise relatedness value of known mother-offspring pairs - 1 standard deviation as relatives in this sample (mean $\pm \mathrm{SD}: 0.42 \pm 0.11$ ), whereas we matched pairs for which the pairwise coefficient of relatedness was lower than the average pairwise relatedness in the population (mean $\pm \mathrm{SD}: 0.02 \pm 0.03$ ) as nonrelatives. Because average female relatedness within troops is relatively high in this species, and there is consequently a lower availability of nonrelated females for each referent individual, we adapted the procedure in baboons so that the judge choose between 2 , rather than among 3 , possible pictures.

\section{Statistical Analysis}

We performed 2-tailed binomial tests for each species to assess whether recognition rates were significantly different than expected by chance, i.e., different from $1 / 2$ for baboons and from $1 / 3$ for the other species. To test the effects of the judge characteristics - their age and sex - we fitted a generalized linear mixed effect model (GLMM), in which we set referent individual and judge identities as crossed random effects to the data (Crawley 2007). The dependent variable was the score of the judge, 0 (failure) or 1 (success), and we chose a quasibinomial error structure to control for overdispersion. The independent variables were the age and the sex of the judges. We then calculated $p$-values via a sample generated after 10,000 simulations from the posterior distribution of the parameters of the fitted model using Markov chain Monte Carlo methods (Bates and Sarkar 2007). To investigate the effect of species, we corrected the recognition rate of each referent individual by both the number of judges (using weighted means) and the rate expected by chance: this gives the deviation of the recognition rate for each individual relative to chance when all judges are taken into account. The distribution of this variable did not deviate from normality (Shapiro-Wilk normality test, $W=0.98, p=0.61$ ), permitting the use of the a posteriori contrasts method for ANOVA model simplification. The contrasts method allows comparison of the mean recognition rate among the various species. After we performed the ANOVA, we grouped the factor levels exhibiting the closest mean recognition rate in a stepwise a posteriori procedure: we tested the significance of the difference between factor levels by comparing models with and without grouped levels (Crawley 2007). We investigated the effect of intraspecific characteristics (age difference between pairs and sex) on individual corrected rates via GLMs (data were available for mandrills, chimpanzees, and baboons). Finally, we performed a Wilcoxon-Mann-Whitney 2-sample test to detect a potential influence of chimpanzee subspecies on facial resemblance detection rate. We conducted all statistical analyses via R (R.2.4.1 2006).

\section{Results}

Can Humans Detect Facial Similarity Between Kin in Nonhuman Primates?

Fifty to 200 judges scored each referent picture (chimpanzees, mean \pm SD: $90.0 \pm 53.1$; gorillas, mean \pm SD: $54.2 \pm 4.3$; mandrills, mean \pm SD: $98.5 \pm 70.5$; baboons, mean \pm SD: 
$84.6 \pm 25.2$ ). Judges recognize related individuals at a rate which exceed the rate expected by chance for 3 species: chimpanzees, gorillas, and mandrills (exact binomial test, $p<0.001$ for each species). This was not the case for baboons, in which the recognition rate was not different from chance expectation (exact binomial test, $p=0.63$ ). The proportion of individuals resembling their relatives more than expected by chance was $100 \%$ for chimpanzees (all pairs), $89 \%$ for gorillas ( 8 of 9 pairs), $60 \%$ for mandrills ( 6 of 10 pairs), and $16 \%$ for baboons ( 2 of 12 pairs). The recognition rates were not sensitive to the characteristics of the judge (simulated $p$ value of the mixed effect model: age effect, $p=0.2$; sex effect, $p=0.9$ ).

\section{Does Primate Species Influence Human Ability to Detect Kin Facial Similarity?}

Human ability to detect kin using facial characteristics did not differ between chimpanzees and gorillas $(F=0.16, \mathrm{df}=1, p=0.69)$. The recognition rate of mandrill facial similarities is similar to the recognition rate of chimpanzees and gorillas pooled together $(F=1.44, \mathrm{df}=1, p=0.22)$. In contrast, the recognition rate for baboon facial similarities is significantly different from the rates of the 3 other species pooled together $(F=4.60, \mathrm{df}=1, p<0.01$; Fig. 2). Thus human judges were successful at detecting facial resemblance between related individuals in 3 primate species, but visual kin recognition performance was not accurate with baboon faces.

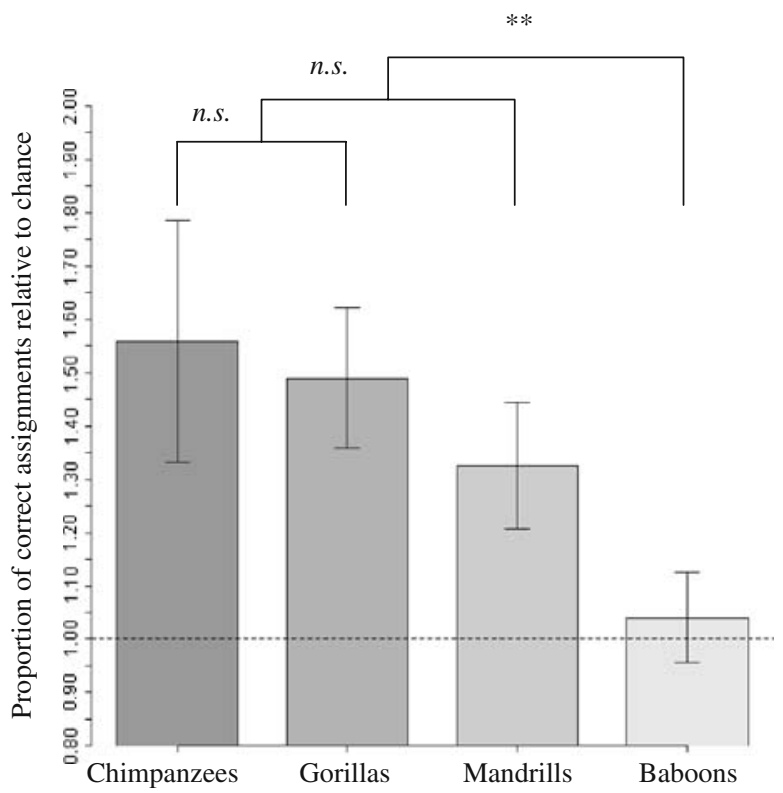

Fig. 2 Facial resemblance between relatives in 4 primate species, according to human judges. The $y$-axis shows the proportion of facial recognition divided by the rate expected by chance. The dashed line represents the recognition rate expected by chance. Facial kin detection rates significantly exceeded the rate expected by chance in chimpanzees, gorillas, and mandrills (exact binomial tests: $p<0.001$ ). In contrast, human judges are not able to detect kin visually in baboons (exact binomial tests: $p=0.63$ ). Error bars represent the standard error of the weighted means. The rate of recognition was not different among chimpanzees, gorillas, and mandrills but baboon scores were significantly different from those of the other species. See text for details. $(* * p<0.01$; non significant (n.s.) $p>0.05$ ). 
Recognition rates for chimpanzees were not influenced by the subspecies tested (Wilcoxon-Mann-Whitney test, $p=0.09$ ).

Does the Age Difference within Pairs and the Sex of Referents Influence the Observed Rates of Recognition?

The mean age difference between pairs was $6.2 \mathrm{yr} \pm 2.0$ for chimpanzees, $3.8 \mathrm{yr} \pm 1.1$ for baboons, and $4.5 \mathrm{yr} \pm 1.4$ for mandrills (comparable information was not available for gorillas). However, age difference did not influence the recognition rate $(F=1.45$, $\mathrm{df}=1, p=0.23$ ). Because all the nonreferent individuals in our protocol were females, the sex of the referent individual determined whether we tested referent individuals against individuals of the same sex, i.e., when the referent individual was a female, or of the other sex, i.e., when the referent individual was a male. The sex of the referent individual was not associated to any significant variation in recognition rates $(F=0.57, \mathrm{df}=1, p=0.45)$.

Is the Judges' Answer Time Related to Performance in Visual Kin Recognition?

The mean answer time of judges was, in seconds, $28.7 \pm 0.7$ (SEM) for chimpanzees,

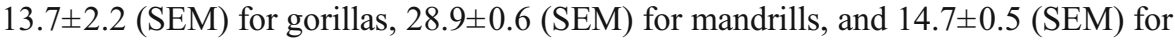
baboons. There is no significant relation between the answer time and the rate of correct facial associations (Spearman correlation coefficient: $r=0.13, \mathrm{df}=38, p=$ $0.59)$.

\section{Discussion}

In an attempt to investigate the extent of the human ability to recognize kin visually, human judges were asked to identify related individuals on the basis of pictures of faces from other primate species. Humans correctly identify kinship on the basis of facial resemblance in chimpanzees, gorillas, and mandrills, but they do not achieve accurate facial kin recognition in baboons.

Our study is the first to show that humans are able to assess facial resemblance in gorillas and mandrills, and replicates the result previously found in chimpanzees (Vokey et al. 2004). The interspecific ability to recognize kin visually observed in nontrained human judges shows that species-specific facial training is not required to detect kin through the face, and suggests that humans are extremely efficient at detecting kin using facial phenotype. Indeed, facial resemblance has an important influence on human social behavior: humans respond to facial resemblance in a way that is consistent with inclusive fitness theory, increasing prosocial behaviors for facesimilar individuals and selectively tempering attributions of attractiveness to other-sex individuals in the context of mating decisions (DeBruine 2002, 2004a, b, 2005; DeBruine et al. 2008, Little et al. 2008, Platek et al. 2003). This supports the view that some highly efficient cognitive abilities designed to perform facial phenotype matching in our own species have evolved through the process of kin selection.

Humans are not able to detect related adult baboons on the basis of the face. The possible effect of the species phylogenetic distance to humans for the performance of 
the human detection system is not a likely explanation for the pattern because we observed accurate visual kin recognition for mandrills despite the fact that the species is as distant to humans as baboons are (Purvis 1995).

It could be proposed that baboon faces appear less attractive to human judges than the other species faces, which may result in a lower level of inspection of baboon faces. However, while judges spent more time looking at mandrills than at baboons ( $c a$. a 2-fold increase), the duration of facial inspection was not related to success in recognition. Thus, although the longer answer time concerning mandrills vs. baboons may reveal a higher attractiveness of mandrill faces vs. baboon faces for human judges, it is unlikely to account for the different kinship recognition rate between the 2 species. Another possibility is that baboons show lower levels of facial variation than other species, making facial-resemblance detection in the species a harder task. A comparative analysis of levels of facial variation may help to answer this question. A last element that might explain the lower performance of humans in baboons is that, in our study, the mean relatedness of individual baboons is measured via pairwise estimates of relatedness derived from genetic markers. Using the TL estimator (Wang 2007), the average relatedness of related pairs used in this study is as high as the average relatedness calculated for mother-offspring pairs that are known from behavioral observations in our study population, thus ensuring a high level of relatedness between individuals used in this study. However, pairwise relatedness estimates calculated using genetic markers are typically less precise than relatedness calculated from pedigree records (Csillery et al. 2006; Wang 2007). Such imprecision can lead to both underestimates and overestimates of relatedness, and if by chance our small sample of related pairs happened to include a disproportionate number of overestimated values, this would reduce the performance of human judges in their correct assignment of kin. Researchers can investigate the possibility further by comparing human performance at visually recognizing kin in baboons when relatedness is known from pedigree records or behavioral observations.

The modification of behavior according to relatedness occurs in various species of nonhuman primate (Chapais and Berman 2004). However, how individuals recognize their kin is still puzzling, in particular when familiarity through association does not necessarily correlate with relatedness. The situation may occur when females pursue multiple mating, inducing males to face paternity uncertainty when making investment decisions. Nevertheless, according to recent studies, fathers seem to know their offspring among several primates living in multimale groups, such as langurs, yellow baboons, and wild chimpanzees (Borries et al. 1999; Buchan et al. 2003; Lehman et al. 2006). For example, male chimpanzees favored their own offspring in some context, i.e., fathers played longer with their offspring but did not groom them preferentially, although the benefits for males and infants associated with such recognition remain to be investigated. There is some evidence that juvenile mandrills are able to discriminate paternal relatives (Charpentier et al. 2007), and discrimination between genetic and nongenetic offspring by males also occurs in baboons (Buchan et al. 2003). In these contexts, it has been proposed that individuals may rely on phenotype matching, i.e., the comparison of a known phenotype to the candidate one, to recognize their kin (Widdig 2007). In chimpanzees, individuals are able to detect facial resemblance between mothers and sons on the basis of photographs, although some researchers have challenged the 
results on methodological grounds (Parr and de Waal 1999). The replication of the result by human judges suggests that chimpanzees and humans share a general mechanism for perception of facial resemblance (Vokey et al. 2004). Our findings indicate that facial resemblance is a reliable indicator of relatedness in at least chimpanzees, gorillas, and mandrills, and future studies should explore whether these primates themselves also share the ability to detect kin facially.

Acknowledgments We thank the city hall of Montpellier for providing an opportunity to recruit judges. We thank all the participants for their collaboration and enthusiasm. We thank J. Benavides and A. Courtiol for suggestions on this manuscript, E. Kerinec and N. Camara for their help in testing, and V. Durand for bibliographic research. Marie J. E. Charpentier is currently supported by a Marie Curie Outgoing fellowship. This article is contribution 2008.081 of the Institut des Sciences de l'Evolution de Montpellier (UMR CNRS 5554).

\section{References}

Altmann, J., Alberts, S. C., Haines, S. A., Dubach, J., Muruthi, P., et al. (1996). Behavior predicts genetic structure in a wild primate group. Proceedings of the National Academy of Sciences of the United States of America, 93, 5797-5801. doi:10.1073/pnas.93.12.5797.

Alvergne, A., Faurie, C., \& Raymond, M. (2007). Differential resemblance of young children to their parents: Who do children look like more. Evolution and Human Behavior, 28, 135-144. doi:10.1016/ j.evolhumbehav.2006.08.008.

Bates, D., \& Sarkar, D. (2007). lme4: Linear mixed-effects models using S4 classes. R package 0.9975-13.

Bergman, T. J., Beehner, J. C., Cheney, D. L., \& Seyfarth, R. M. (2003). Hierarchical classification by rank and kinship in baboons. Science, 302, 1234-1236. doi:10.1126/science.1087513.

Borries, C., Launhardt, K., Epplen, C., Epplen, J., \& Winkler, O. (1999). Males as infant protectors in Hanuman langurs (Presbytis entellus) living in multimale groups: Defence pattern, paternity and sexual behavior. Behavioral Ecology and Sociobiology, 46, 350-356. doi:10.1007/s002650050629.

Buchan, J. C., Alberts, S. C. Silk, J. B., Altmann, J. (2003). True paternal care in a multi-male primate society. Nature, 425(6954), 179-181.

Caillaud, D., Levréro, F., Cristescu, R., Gatti, S., Dewas, M., et al. (2006). Gorilla susceptibility to Ebola virus: The cost of sociality. Current Biology, 16, R489-R491. doi:10.1016/j.cub.2006.06.017.

Chapais, B., \& Berman, C. M. (2004). Kinship and behavior in primates. Oxford: Oxford University Press.

Charpentier, M., Peignot, P., Hossaert-Mckey, M., \& Wickings, E. J. (2007). Kin discrimination in juvenile mandrills, Mandrillus sphinx. Animal Behaviour, 73, 37-45. doi:10.1016/j.anbehav. 2006.02.026.

Cheney, D. L., \& Seyfarth, R. M. (1982). Vocal recognition in free-ranging vervet monkeys. Animal Behaviour, 28, 362-367. doi:10.1016/S0003-3472(80)80044-3.

Cheney, D. L., \& Seyfarth, R. M. (1999). Recognition of other individuals' social relationships by female baboons. Animal Behaviour, 58, 67-75. doi:10.1006/anbe.1999.1131.

Cowlishaw, G. (1999). Ecological and social determinants of spacing behaviour in desert baboon groups. Behavioral Ecology and Sociobiology, 45, 67-77. doi:10.1007/s002650050540.

Crawley, M. J. (2007). The R book. Chichester: John Wiley \& Sons.

Csillery, K., Johnson, T., Beraldi, D., Clutton-Brock, T., Coltman, D., et al. (2006). Performance of marker-based relatedness estimators in natural populations of outbred vertebrates. Genetics, 173, 2091-2101. doi:10.1534/genetics.106.057331.

Dasser, V. (1987). Slides of group members as representations of the real animals (Macaca fascicularis). Ethology, 76, 65-73.

Dasser, V. (1988). A social concept in Java monkeys. Animal Behaviour, 36, 225-230. doi:10.1016/ S0003-3472(88)80265-3.

DeBruine, L. M. (2002). Facial resemblance enhances trust. Proceedings of the Royal Society of London. Series B. Biological Sciences, 269, 1307-1312. doi:10.1098/rspb.2002.2034.

DeBruine, L. M. (2004a). Facial resemblance increases the attractiveness of same-sex faces more than other-sex faces. Proceedings of the Royal Society of London. Series B. Biological Sciences, 7, 20852090. doi:10.1098/rspb.2004.2824. 
DeBruine, L. M. (2004b). Resemblance to self increases the appeal of child faces to both men and women. Evolution and Human Behavior, 25, 142-154. doi:10.1016/j.evolhumbehav.2004.03.003.

DeBruine, L. M. (2005). Trustworthy but not lust-worthy: Context-specific effects of facial resemblance. Proceedings of the Royal Society of London. Series B. Biological Sciences, 272, 919-922. doi:10.1098/rspb.2004.3003.

DeBruine, L. M., Jones, B. C., Little, A. C., \& Perrett, D. I. (2008). Social perception of facial resemblance in humans. Archives of Sexual Behavior, 37, 64-77. doi:10.1007/s10508-007-9266-0.

Dufour, V., Coleman, M., Campbell, O., Petit, O., \& Pascalis, O. (2004). On the species-specificity of face recognition in human adults. Current Psychology of Cognition, 22, 315-333.

Dufour, V., Pascalis, O., \& Petit, O. (2006). Face processing limitation to own species in primates: A comparative study in brown capuchins, Tonkean macaques and humans. Behavioural Processes, 73, 107-113. doi:10.1016/j.beproc.2006.04.006.

Johnson, M. H., Dziurawiec, S., Ellis, H., \& Morton, J. (1991). Newborns’ preferential tracking of facelike stimuli and its subsequent decline. Cognition, 40, 1-19. doi:10.1016/0010-0277(91)90045-6.

Kahumbu, P., \& Eley, R. M. (1991). Teeth emergence in wild live baboons in Kenya and formulation of a dental schedule for aging wild baboon populations. American Journal of Primatology, 23, 1-9. doi:10.1002/ajp.1350230102.

Kelly, D. J., Quinn, P. C., Slater, A. M., Lee, K., Ge, L., \& Pascalis, O. (2007). The other-race effect develops during infancy: Evidence of perceptual narrowing. Psychological Science, 18, 1084-1089. doi:10.1111/j.1467-9280.2007.02029.x.

Lehmann, J., Fickenscher, G., \& Boesch, C. (2006). Kin biased investment in wild chimpanzees. Behaviour, 143, 931-955. doi:10.1163/156853906778623635.

Lindsay, D. S., Jack, P. C., \& Christian, M. A. (1991). Other-race face perception. The Journal of Applied Psychology, 76, 587-589. doi:10.1037/0021-9010.76.4.587.

Little, A. C., Burriss, R. P., Jones, B. C., DeBruine, L. M., \& Caldwell, C. A. (2008). Social influence in human face preference: Men and women are influenced more for long-term than short-term attractiveness decisions. Evolution and Human Behavior, 29, 140-146. doi:10.1016/j.evolhumbehav. 2007.11.007.

Maloney, L. T., \& Dal Martello, M. F. (2006). Kin recognition and the perceived facial similarity of children. Journal of Vision (Charlottesville, Va.), 6, 1047-1056. doi:10.1167/6.10.4.

Niedenthal, P. M., Halberstadt, J. B., Margolin, J., \& Innes-Ker, E. S. H. (2000). Emotional state and the detection of change in facial expression of emotion. European Journal of Social Psychology, 30, 211222. doi:10.1002/(SICI)1099-0992(200003/04)30:2<211::AID-EJSP988>3.0.CO;2-3.

O'Toole, A. J., Deffenbacher, K. A., Valentin, D., \& Abdi, H. (1994). Structural aspects of face recognition and the other-race effect. Memory \& Cognition, 22, 208-224.

Parr, L. A., \& de Waal, F. B. M. (1999). Visual kin recognition. Nature, 399, 647-648. doi:10.1038/21345.

Parr, L. A., Dove, T., \& Hopkins, W. D. (1998). Why faces may be special: Evidence of the inversion effect in chimpanzees. Journal of Cognitive Neuroscience, 10, 615-622. doi:10.1162/089892 998563013.

Parr, L., Winslow, J. T., \& Hopkins, W. D. (1999). Is the inversion effect in rhesus monkeys face-specific? Animal Cognition, 2(3), 123-129.

Pascalis, O., \& Bachevalier, J. (1998). Face recognition in primates: A cross-species study. Behavioural Processes, 43, 87-96. doi:10.1016/S0376-6357(97)00090-9.

Phillips-Conroy, J. E., Bergman, T., \& Jolly, C. J. I. (2000). Quantitative assessment of occlusal wear and age estimation in Ethiopian and Tanzanian baboons. In P. F. Whitehead, \& C. J. Jolly (Eds.), Old world monkeys (pp. 321-340). Cambridge, UK: Cambridge University Press.

Platek, S. M., Critton, S. R., Burch, R. L., Frederick, D. A., Myers, T. E., \& Gallup, G. G. (2003). How much paternal resemblance is enough? Sex differences in hypothetical decisions but not in the detection of resemblance. Evolution and Human Behavior, 24, 81-87. doi:10.1016/S1090-5138(02)00117-4.

Purvis, A. (1995). A composite estimate of primate phylogeny. Philosophical Transactions of the Royal Society of London Series B, 348, 405-421. doi:10.1098/rstb.1995.0078.

Rendall, D., Rodman, P. S., \& Emond, R. E. (1996). Vocal recognition of individuals and kin in freeranging rhesus monkeys. Animal Behaviour, 51, 1007-1015. doi:10.1006/anbe.1996.0103.

R.2.4.1. (2006). The $R$ development core team.

Sangrigoli, S., Pallier, C., Argenti, A. M., Ventureyra, V. A. G., \& de Schonen, S. (2005). Reversibility of the other-race effect in face recognition during childhood. Psychological Science, 16, 440-444.

Sugita, Y. (2008). Face perception in monkeys reared with no exposure to faces. Proceedings of the National Academy of Sciences of the United States of America, 105, 394-398. doi:10.1073/ pnas.0706079105. 
Tanaka, M. (2003). Visual preference by chimpanzees (Pan troglodytes) for photos of primates measured by a free choice-order task: Implication for influence of social experience. Primates, 44, 157-165.

Tomonaga, M. (1994). How laboratory-raised Japanese monkeys (Macaca fuscata) perceive rotated photographs of monkeys: Evidence for an inversion effect in face perception. Primates, 35, 155-165. doi:10.1007/BF02382051.

Vokey, J. R., Rendall, D., Parr, L. A., de Waal, F. B. M., \& Tangen, J. M. (2004). Visual kin recognition and family resemblance in chimpanzees. Journal of Comparative Psychology, 118, 194-199. doi:10.1037/0735-7036.118.2.194.

Wang, J. (2007). Triadic IBD coefficients and applications to estimating pairwise relatedness. Genetical Research, 89, 135-153. doi:10.1017/S0016672307008798.

Widdig, A. (2007). Paternal kin discrimination: The evidence and likely mechanisms. Biological Reviews of the Cambridge Philosophical Society, 82, 319-334. doi:10.1111/j.1469-185X.2007.00011.x.

Wittig, R. M., Crockford, C., Seyfarth, R. M., \& Cheney, D. L. (2007). Vocal alliances in Chacma baboons (Papio hamadryas ursinus). Behavioral Ecology and Sociobiology, 61, 6. doi:10.1007/s00265-0060319-5. 